\title{
UTILISATION OF LASER SCANNING TECHNOLOGY AND DIGITAL IMAGES FOR MEASUREMENTS OF INDUSTRIAL OBJECTS - A CASE STUDY
}

\author{
Dorota Zawieska, Jakub Markiewicz \\ Faculty of Geodesy and Cartography, Warsaw University of Technology \\ Warsaw, Poland
}

\begin{abstract}
Projection of a complicated geometry of industrial objects is the complex issue, which requires properly planned and prepared measurements. Such objects must be accurately inventoried, but their complicated nature often makes the access and the visibility of their entire surface very difficult. Documentation of measurements is often prepared in the form of sketches, plans or maps, which are amended with photographic documentation. The objective of this paper is to test the possibilities to apply laser scanning and the network of digital images for inventory and monitoring of technical conditions of industrial objects. Processing of a precise documentation acquired basing on terrestrial laser scanning data or dense points clouds generated from digital images still causes many difficulties and problems. Although data processing algorithms have been intensively developed with respect to generation of high resolution orthoimages or precise vector drawings, the existing problems are still connected with limitations related to imperfections of both techniques of measurements.
\end{abstract}

Keywords: photogrammetry, computer vision (CV), SfM, terrestrial laser scanning, engineering surveys, deformations

\section{Introduction}

Projection of appearance and geometry of complicated industrial objects is a complex issue, which requires implementation of planned and prepared measurements. Such objects must be inventories with the high accuracy, but their complicated nature often makes the visibility and access to entire objects difficult. Developed documentation was often prepared in the form of sketches, plans or maps, which were amended with photographic documentation. Projection of objects in such forms is often insufficient. Due to the increasing number of industrial objects and the technological development the demand to modify the methods of inventory 
appeared. Designing new elements, with the use of CAD/CAM systems requires that objects are described as 3D forms. Measurements were initially performed using tacheometers, allowing for obtaining spatial co-ordinates of every measured point. This technique required the use of a reflector and involvement of two persons. In the case of an industrial plant, zones inaccessible for humans often occur; therefore reflector-less tacheometers appeared on the market, allowing for contactless measurements. However, measurements lasted too long and they were not efficient.

Basing on this technology, a laser scanner was constructed, which performs automatic measurements of millions points and operates faster than a total-stations.

Terrestrial laser scanning produces a three-dimensional description of geometry of a scanned object. The accuracy of measurements does not differ in this case from the accuracy performed using precise tacheometers; the speed of measurements reaches about one million points per second. Integration of terrestrial laser scanning with digital photography allows for creation of a realistic three-dimensional model. Data acquired with the use of a laser scanner may be used for inventory of complicated industrial objects and for valuable analyses which could not be performed using conventional techniques (Elseberg, et. al, 2014). Operations with the "point cloud" concerns realistic visualisation, precise measurements in a space transferred to the virtual reality and they are also the basis for advanced spatial modelling and reverse engineering (Gergont, Kierys, 2009, Luhman, et. al, 2013, Vosselman \& Mass, 2012). Technology of terrestrial laser scanning aims at the full automation, but complete utilisation of acquired geometrical data requires specialist knowledge and appropriate software tools.

Together with the development of measuring methods based on the terrestrial laser scanning, algorithms and methods of point clouds generation, basing on digital images applied in computer vision (CV) and photogrammetry, were also developed (Braazetti \& Scaioni, 2009, Luhman, et. al, 2013). One of the most modern approaches to point cloud generation is the method based on the structure-frommotion (SfM) algorithms, which are widely applied in computer vision algorithms. It is the fully automated method which allows for implementation of relative orientation of photographs, generation of dense point clouds basing on the network of photographs only. Its advantage concerns the ability to process both metric and non-metric photographs, which do not have the camera calibration certificate (Markiewicz \& Turek, 2014; Mousa, 2014).

\section{Factors which influence the accuracy of laser scanning}

Before the measurements with the use of terrestrial laser scanning were started a series of laboratory tests were performed, which aimed at the analysis of the influence of various factors on the scanning accuracy (Radziejewski, 2013).

\subsection{Evaluation of the reflectance on surfaces of industrial objects}

The experiment concerning scanning of a pattern of a full colour palette and a palette of black shades was performed using the Z+F 5006h scanner. Performed laboratory tests proved that the intensity of reflectance of a laser scanner signal is similar to natural visual sensing. The darker the scanned colour, the lower the intensity of reflectance. White objects will be best projected, and projection of black objects will be the worst. Figure 1 presents the original pattern of colours with the partially 
overlapping resulting scanned image of the pattern of colours (various shades of grey).

Another issue is related to the scanned object surface colour, which influences the accuracy of measurements. If the object colour is darker then the lower reflectance intensity is (Fig.2). It influence on the worse accuracy of measurements The manufacturer of the Z+F IMAGES 5006h laser scanner specifies that the reflectance from the black surface results in the accuracy of measurements which is three times worse than the reflectance from a white surface.

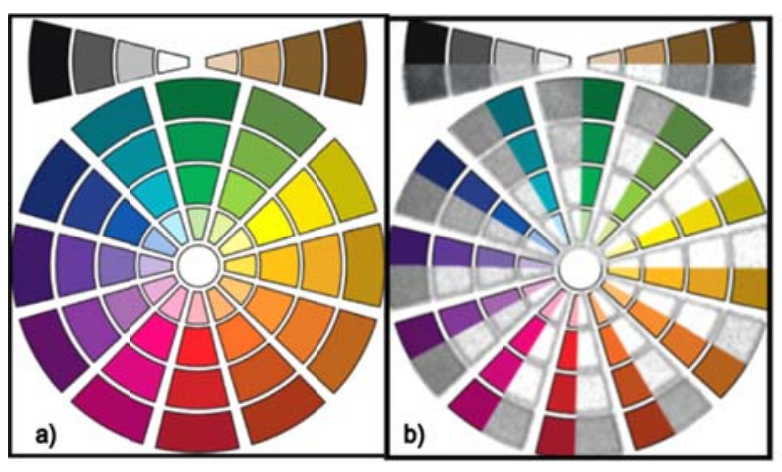

Fig. 1. Intensity of reflectance of a colour palette of a beam of shades

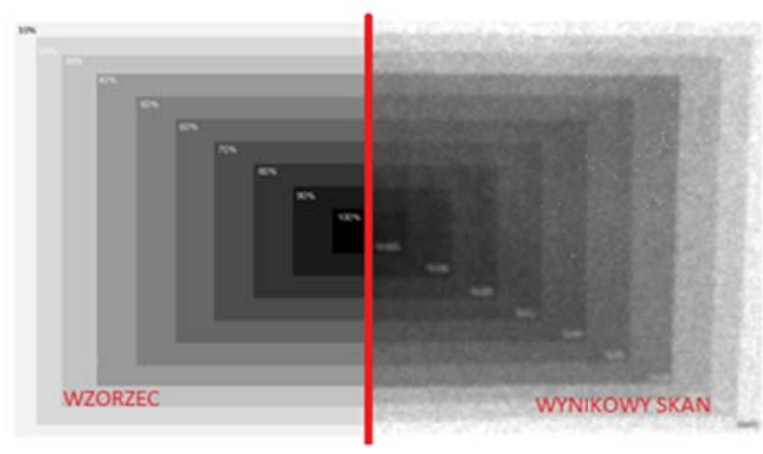

Fig. 2. Intensity of reflectance of black (50\% pattern/ $50 \%$ scan)

\subsection{Assessment of the influence of reflectance of pipe elements}

The next important experiment concerned the evaluation of the impact of reflectance of pipe elements on their projection in the point cloud. Excluding water pipelines, elements which are characterised by the cylindrical or spherical geometry, become the most difficult surfaces for scanning (Fig. 3). Projection of surfaces of pipes is characterised by the high jumps of the intensity, since the signal which is reflected from a surface perpendicular to the instrument results in the highest value of the reflectance intensity. This intensity of reflectance is decreased when this angle of deflected from the right angle.

Figure 4 presents results of laboratory testing, which comprised scanning of various industrial items and testing the intensity of reflectance of a scanner beam from particular objects. White surfaces on the scanned image project items of the highest reflectance intensity. It has been observed that a PVC pipe is characterised by the higher reflectance intensity than a steel or an aluminium pipe. 


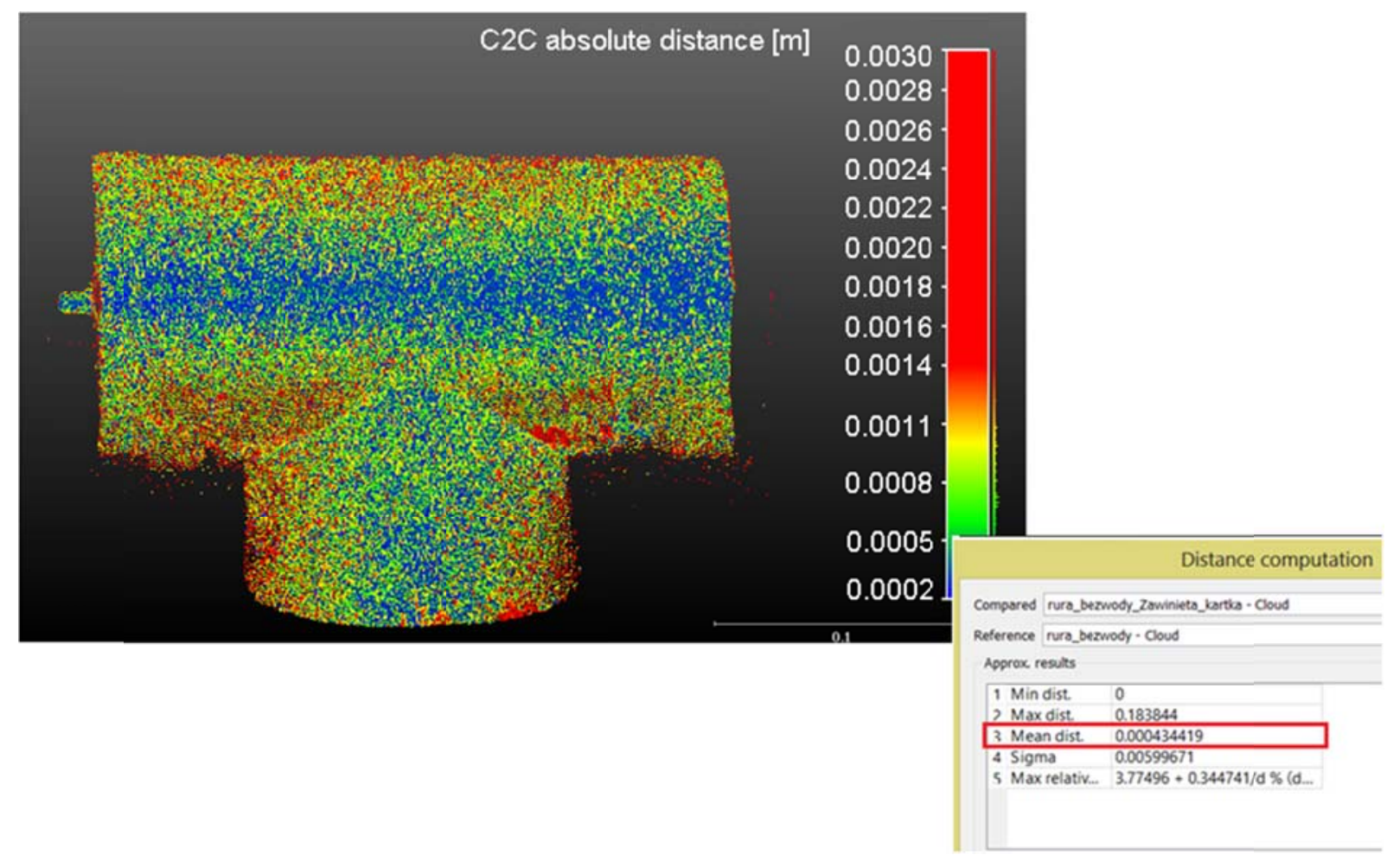

Fig. 3. The accuracy analysis of scanning of an industrial item (comparison of two point clouds from the same scanner station in the CloudCompare tool; the scope of deviations from $0.0002 \mathrm{~mm}$ (blue ) to $0.003 \mathrm{~mm}$ (red))
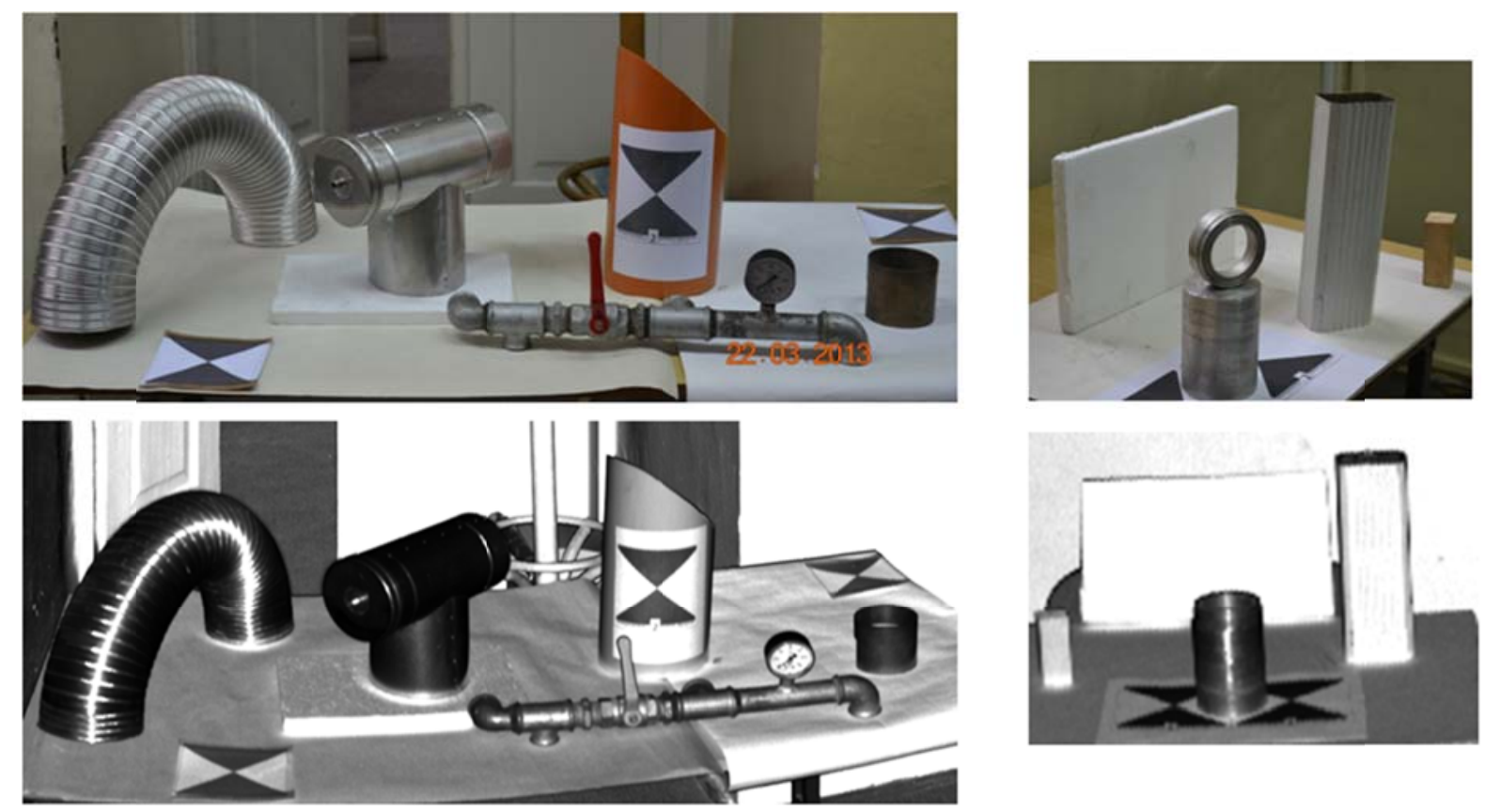

Fig.4. The digital photograph and the scanned image of various surfaces of industrial items 


\subsection{Analysis of the influence of atmospheric conditions}

Another experiment concerned the analysis of the influence of atmospheric conditions (water drops) occurring on the scanned objects on the accuracy of its projection. Measurements performed using the laser scanner are often on an open area; due to diversified atmospheric conditions it was simulated that the measurements took place just after the rainfall. In order to obtain a similar effect a sprinkler of water was used. The entire surface of a steel pipe was intensively covered with water - a thin water layer and many big drops of water were generated. Two sessions of measurements were performed using the laser scanner: for the dry and for the wet pipe; then both point clouds were compared using the CloudCompare software tool (Fig.5). The average difference between point clouds is much higher than the differences between the same scans of a dry pipe and it equals to $2.0 \mathrm{~mm}$. Water occurring on the surface of the scanned object shortens the distance range and influence on accuracy of measurements. In Fig.5 compared rays, which are presented as red, are "sliding" on the surface under the influence of water - some transmitted signal beams do not return to the scanner. It may be also noticed that some concentrations of water drops are projected in the scanned image.

Blue areas in Fig. 5 present the differences of the order of $0.2 \mathrm{~mm}$, the green areas correspond to differences of the order of $0.5 \mathrm{~mm}$; the yellow areas present the differences of the $0.9 \mathrm{~mm}$ order. Remaining differences, bigger than $1.2 \mathrm{~mm}$ are presented in red.

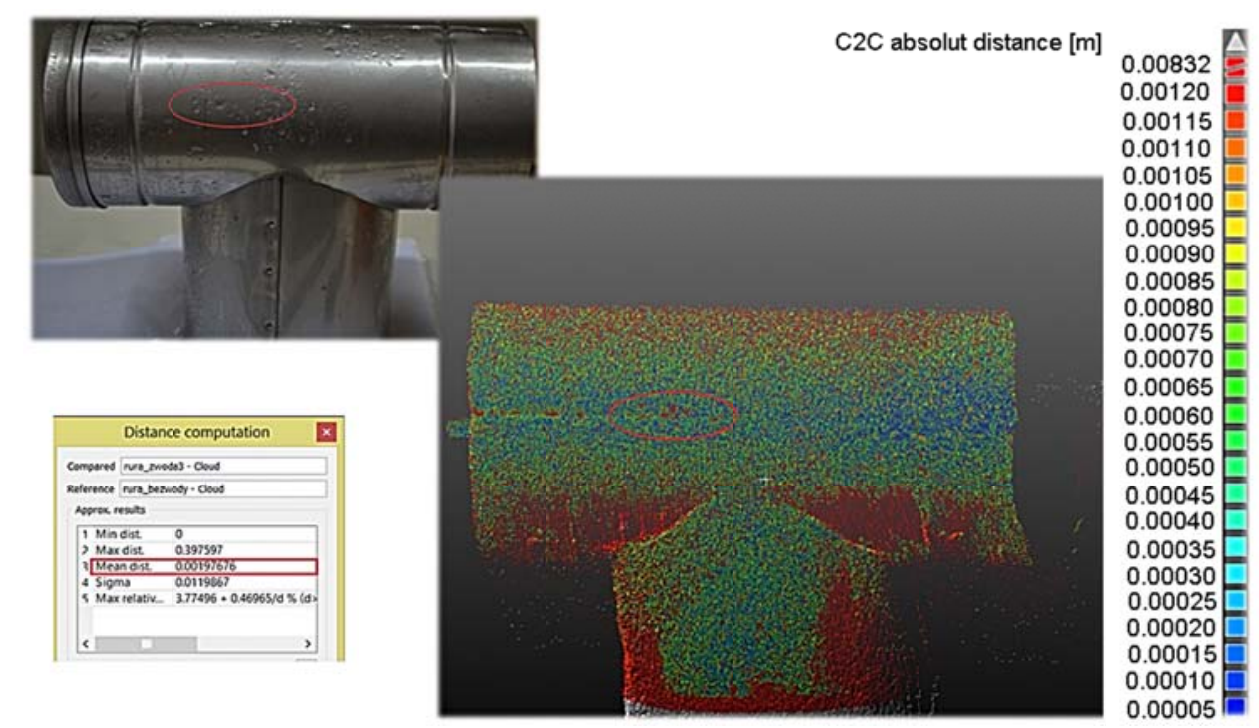

Fig. 5. A digital photograph of a wet pipe and graphical presentation of the influence of water on the accuracy of measurements of a scanned element

\subsection{Analysis of the influence of the angle of scanning of an industrial surface}

Industrial installations are characterised by cylindrical or spherical shapes, and often, by the shining surface of low roughness. A good example is a steel pipe; its resulting scanned image deviates from a real shape. The surface directed perpendicularly to the laser beam generates too extensive signal reflectance what results in deformations of the real shape of the cylindrical pipe (Fig.6). Those points are 
characterised by the measured distance from the scanner, which is longer than the real value (they are projected behind the object). Another area of error generation are the pipe edges - surfaces which are inclined under the small angle, where the earlier "sliding" effect of the laser beam can also be noticed. Such points are projected before the scanned object. i.e. to the contrary in relation to the points of the surface which is perpendicularly directed towards the laser beam.

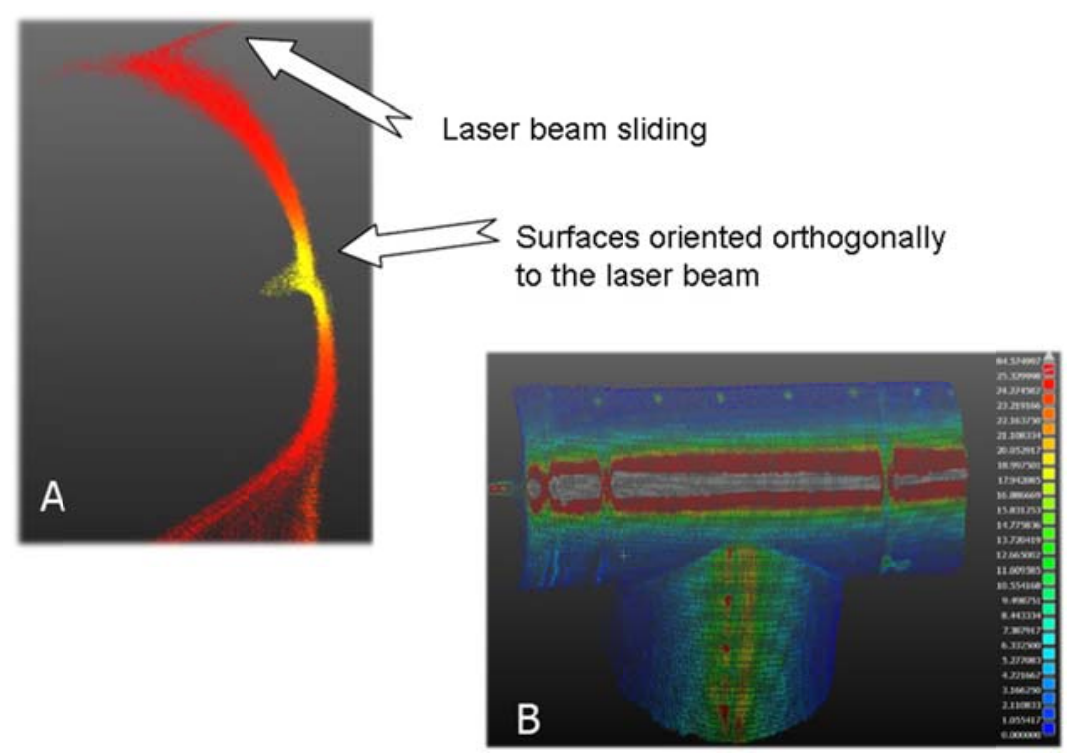

Fig.6. Intensity of reflectance of a scanned pipe (A) - a pipe profile in the point cloud, yellow - too intensive beam reflectance; $(B)$ - intensity of reflectance of a scanned pipe, grey - too intensive reflectance

\section{Characteristics of an analysed objects and performed field works}

The tank is located in a basin of the height of approx. $3 \mathrm{~m}$ with a surrounding bank, being an ideal place for placing measuring stations (due to the visibility). Dimensions of the reservoir are: its diameter $23.8 \mathrm{~m}$, and height $12.0 \mathrm{~m}$.

Pipeline installations are placed around the analysed object; their diameters do not exceed $0.40 \mathrm{~m}$ and the length of each pipe does not exceed approx. $50 \mathrm{~m}$ within a selected area. The measured shell of the fuel tank consists of 7 levels which are connected by welds. They are good reference points for matching (relative orientation) scans; therefore it is necessary to apply such scanning resolution which would allow for explicit identification of welds. Considering that banks are located within the distance of $10 \mathrm{~m}$ from the tank, the scanning resolution of the order of $6 \mathrm{~mm}$ per $10 \mathrm{~m}$ has been selected for particular laser stations; these stations were placed in such a way which would allow for measuring the tank from all sides, in order to eliminate the, so-called, blind spots (Fig.7). 
A

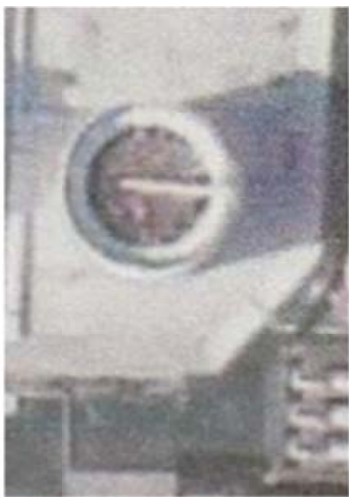

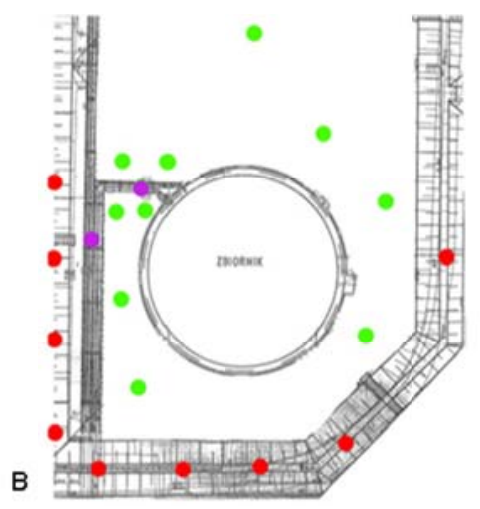

C

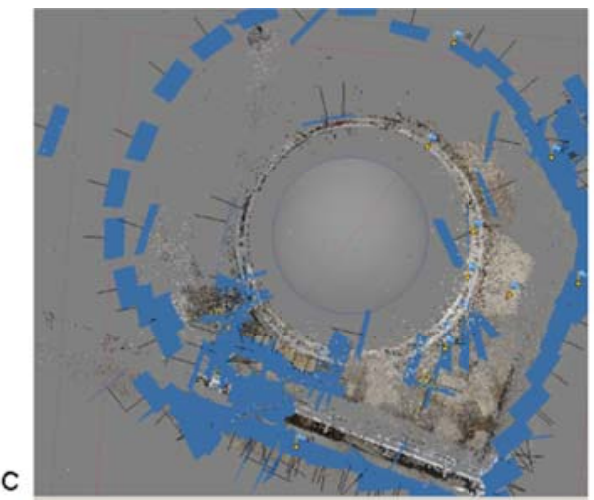

Fig. 7. (A) and airborne photograph of a tank (Radziejewski et al., 2014);

(B) A sketch of positions of the Z+F 5006 scanner stations (Radziejewski et al., 2014);

(C) A sketch of position of the camera stations

24 self-adhesive targets and 5 tripods with rotating targets were placed on the scanned object. During measurements the day was cloudy, without rainfalls. On the ground was lying partially and fast melting snow (Radziejewski et al., 2014).

Experiments were performed using the terrestrial Z+F Imager 5006h scanner and the terrestrial Canon 5D Mark II camera of a fragment of the fuel terminal, consisting of a fuel tank (Fig.7), pipelines and other installations. This allowed for generation of $3 \mathrm{D}$ visualisation of the tank, modelling of pipes and testing deformations and the accuracy of projected surfaces.

\section{Analysis of acquired point clouds}

Before further processing point clouds acquired from the terrestrial laser scanning should be cleaned from noises, which occurred due to the influence of atmospheric conditions. They covered the entire surface of the tank; as a result of the big number of the laser stations the existing blind spots could be eliminated (Fig. 8). The terrestrial laser scanning method is the best method of measurements of such objects, since the object surface is not highly diversified in colours or in texture. The average resolution of the point cloud equalled to $3 \mathrm{~mm}$ (Radziejewski et al., 2014).
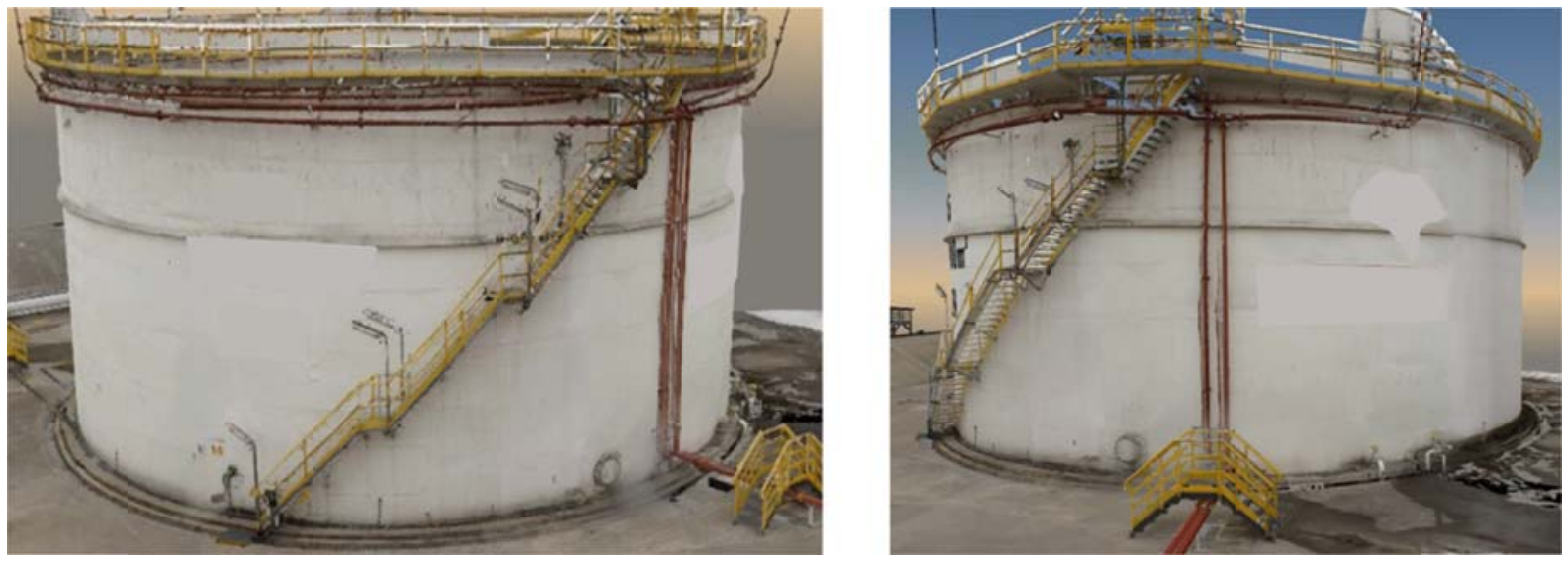

Fig.8. A point cloud acquired from terrestrial laser scanning 
AgiSoft software was used for generation of the point cloud basing on the dense matching of digital photographs. Photographs taken with an external camera, Canon 5D Mark II, at the time of scanning, were used for this purpose. Fig. 7c presents a sketch of distribution of the camera stations. It may be seen that the higher number of stations could be located in the front part of the tank. The existing embankment allowed for taking images at two levels in the front part of the tank. Points used for the needs of orientation of data from the terrestrial laser scanning were also used in the case of taking photographs. The final effect is presented in Fig.9.
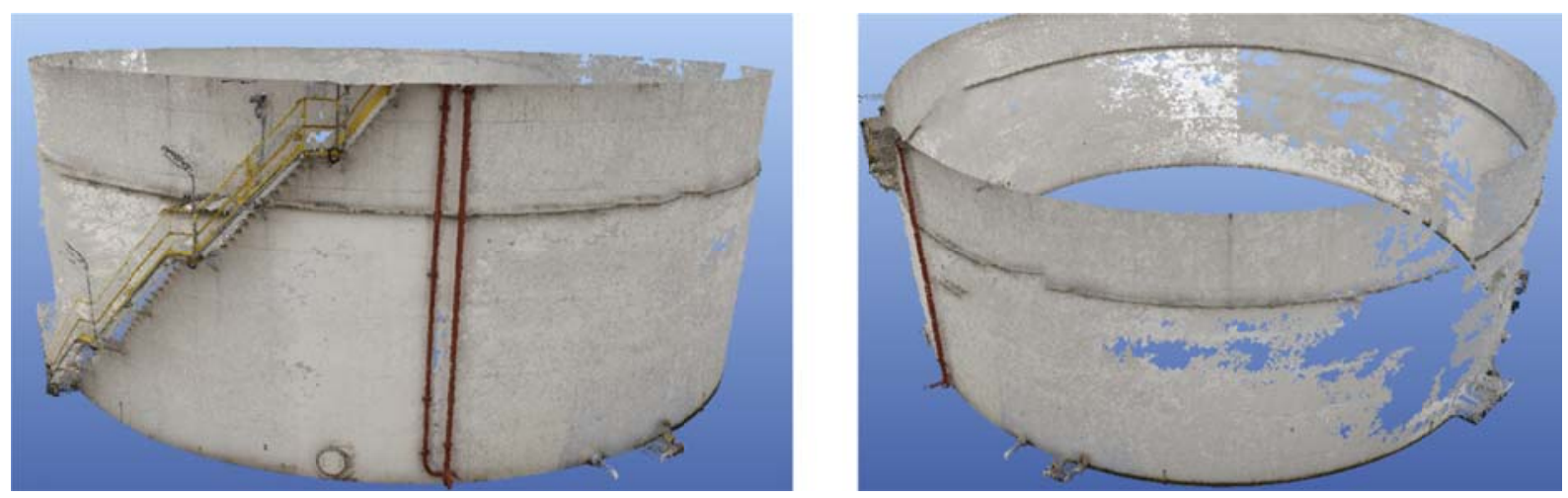

Fig.9. A point cloud acquired from the dense matching (resolution of $4 \mathrm{~mm}$ )

The object was characterised by a uniform texture; unfortunately this resulted in blind spots (lack of data) which existed in places where images were acquired with less overlap and from the lower number of camera stations (Fig.9). The average resolution of the generated point cloud equalled to $4 \mathrm{~mm}$.

In order to test the possibility to project shapes of industrial objects (transmitting pipes; Fig.10), the accuracy which could be achieved using both measuring techniques, was tested. The point cloud acquired from the terrestrial laser scanning is characterised by lower noise comparing to the cloud obtained from dense matching.

A reference cylinder was assumed as the reference shape; it was fitted into the existing data set. It was the basis for generation of the orthoimage; this allowed for determination of deviations from the reference plane (Fig.11). In the case of the point cloud from the terrestrial laser scanning the deviations were included within the range $+/-5 \mathrm{~mm}$; deviations resulting from the dense matching - within the range $+/-11 \mathrm{~mm}$.
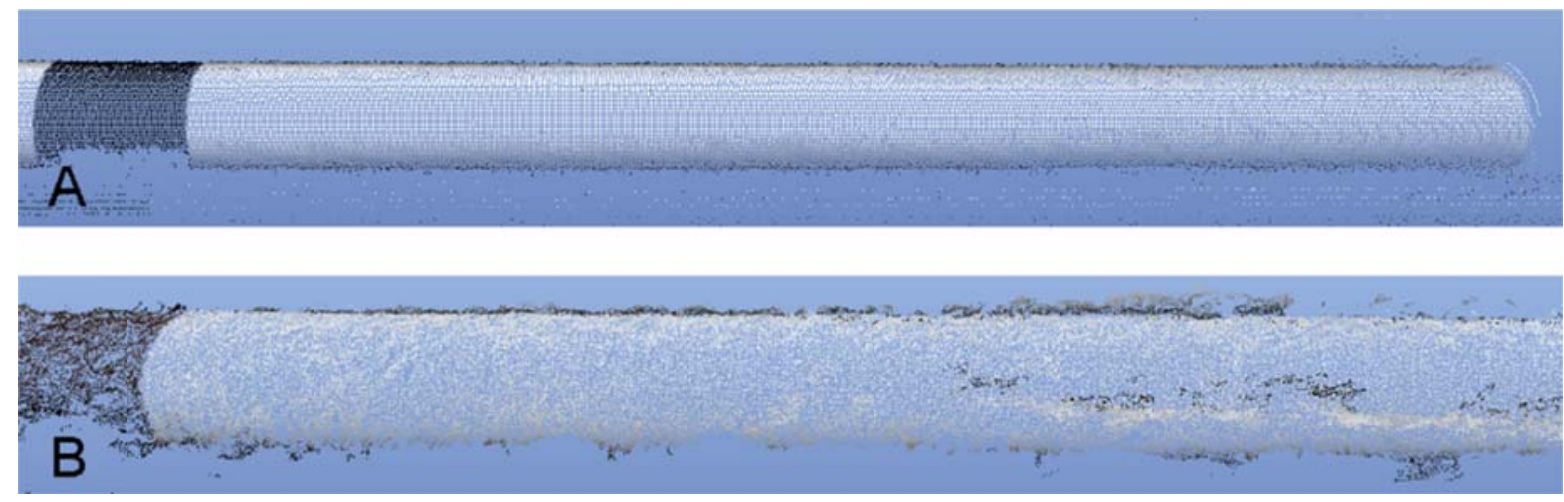

Fig.10. 3D view of the point cloud of a pipe: A - from terrestrial laser scanning,

$B$ - from the dense matching 


\section{A}

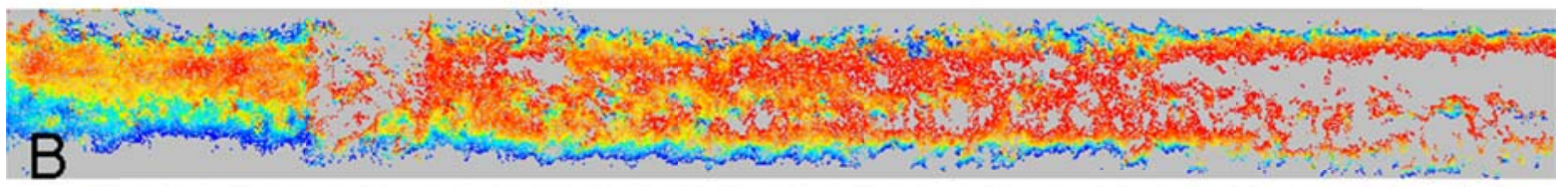

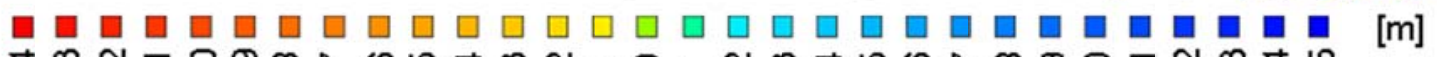

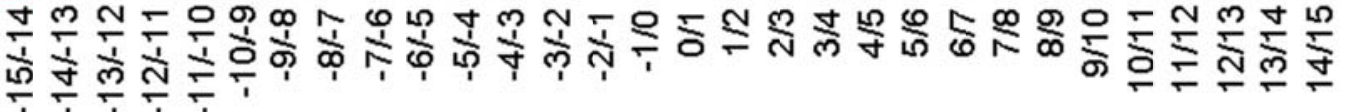

Fig.11. A map of deviations on points from the theoretical shape of a pipe: A - from terrestrial laser scanning, B - from the dense matching

\section{Analysis of the tank deformations}

Another important step in performed analyses concerned the tests of deformations of the tank acquired with the use of two methods: from automatic matching of digital images and from terrestrial laser scanning data. Preliminary accuracy analysis resulting from the terrestrial laser scanning was earlier presented in the publication (Radziejewski et al., 2014), where the authors compared results obtained from the laser scanning with conventional tacheometric measurements. At present, where the usefulness of automatic matching of digital images is tested, data firom the terrestrial laser scanning is considered as reference data.

As a result of performed analyses it may be noticed that, in the case of the terrestrial laser scanning data, the mean error of deviations from the plane of an ideal cylinder equals to $34 \mathrm{~mm}$; in the case of dense image matching it achieves the value of $37.5 \mathrm{~mm}$. The distribution of errors is also similar and existing differences are only the result of the fact that places where deviations occurred were occluded by the infrastructure existing around the tank.
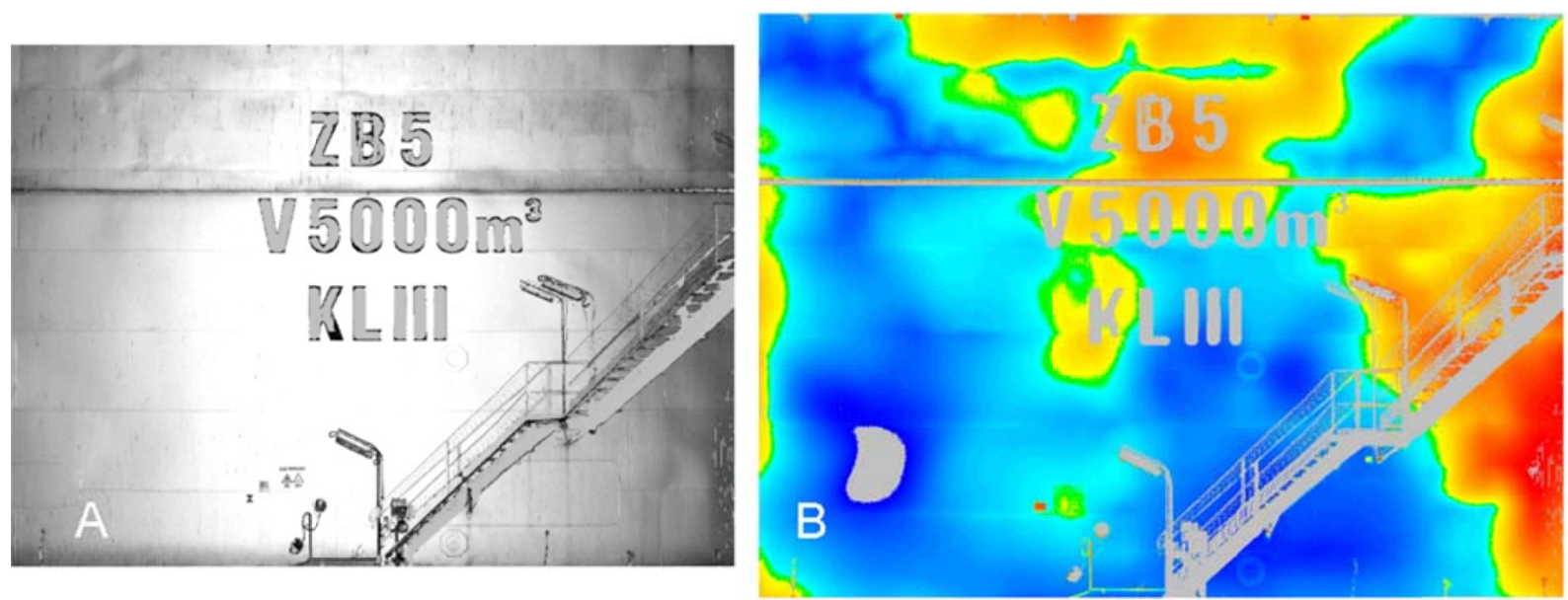

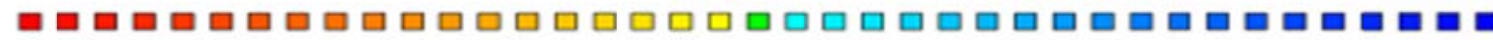

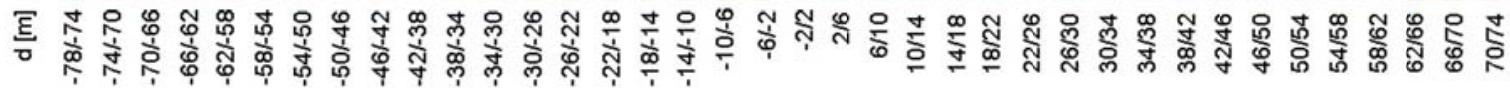

Fig. 12. (A) The orthoimage of a fragment of the analysed tank (TLS),

(B) A map of deviations of points from the theoretical surface (TLS) 

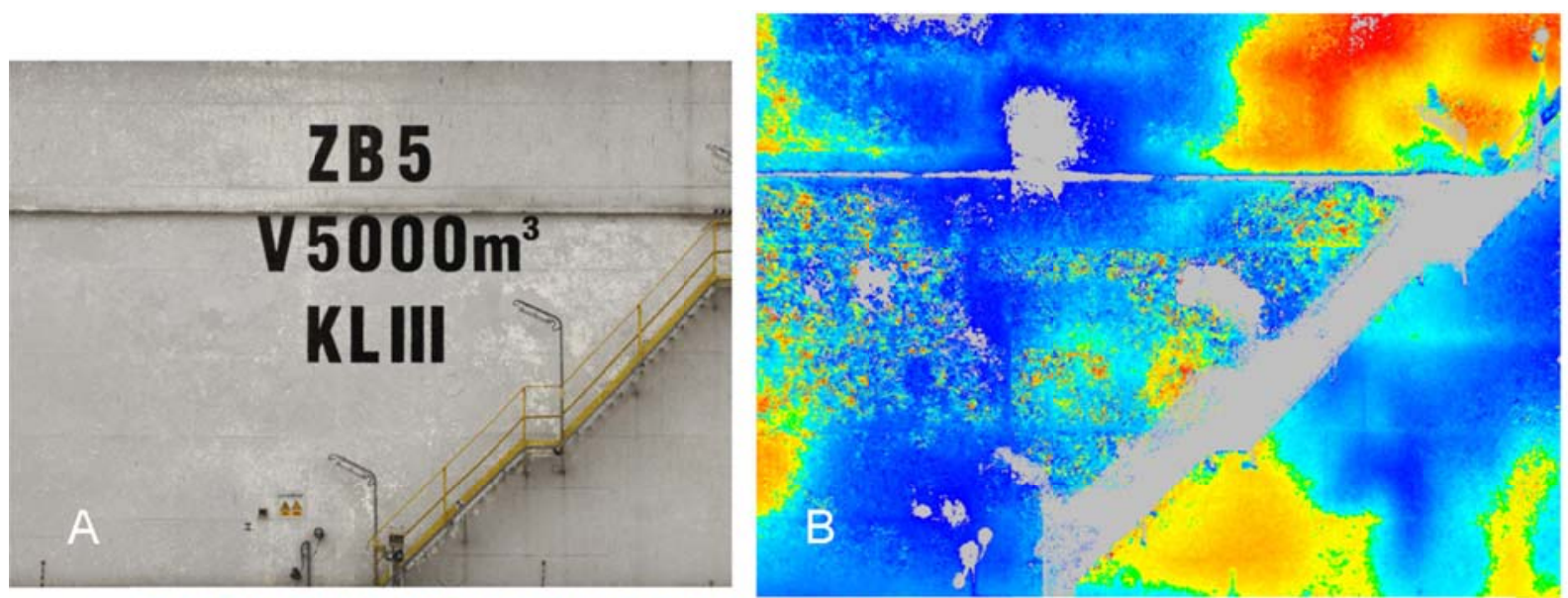

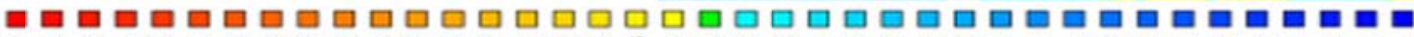

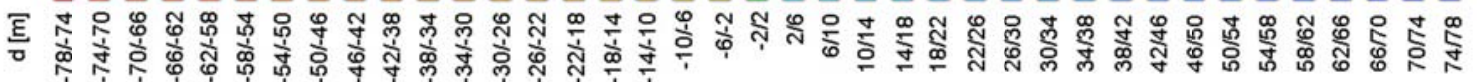

Fig. 13. (A) The orthoimage of a fragment of the analysed tank (dense matching), (B) The map of deviations of points from the theoretical surface (dense matching)

\section{Concluding remarks}

Concluding the performed experiments and analyses attention should be paid to many aspects concerning utilisation of the laser scanning technology and photogrammetric methods in measurements of industrial objects.

During the initial experiments, tests of the accuracy of measurements of industrial pipes performed using the terrestrial laser scanner together with the evaluation of the influence of reflectance of scanned surfaces. The accuracy of projection of white scanned surfaces is three times higher than in the case of darker surfaces. Due to the fact that the scanner signal is not reflected by ideal black surfaces, scanning such surfaces may partially lead to the lack of data in such places. Experiments concerning testing of pipes confirmed that it is difficult to correctly project these objects using terrestrial laser scanning technique. Steel pipes are characterised by the low roughness and shining surfaces and resulting point clouds partially deviate from the reality. The mean error of measurements of the double-scanned steel pipe equals to $0.4 \mathrm{~mm}$. Water drops present on the pipe results in errors of measurement which are 5 times bigger. Bigger drops of water are projected in scanned images.

While processing large industrial objects using photogrammetric methods the necessity to plan the photogrammetric control and distribution of camera stations should be considered. In the case of objects of uniform texture lower accuracy of projection of shapes should be expected (comparing to measurements performed with the use of the terrestrial laser scanner), but they are still sufficient for engineering works.

As a result of performed experiments it may be stated that both methods allow for achieving the high accuracy of projection of shapes of industrial objects and sometimes they even complement those shapes. It should be also considered that factors which influence the accuracy of the shape projection include atmospheric conditions, the structure and texture of investigated objects as well as the geometry of distribution of stations of measuring sensors. 


\title{
Reference
}

Brazetti, L., \& Scaioni M. (2009). Automatic orientation of image sequences for 3D object reconstruction: first results of a method integrating photogrammetric and computer vision algorithms. International Archives of Photogram., Remote Sensing, Volume XXXVIII-5/W1

Elseberg J., Borrmann .D., Schauer J., Nuchter A., Koriath D., and Rautenberg U., (2014) A sensor skid for precise 3d modeling of production lines. ISPRS Annals of the Photogrammetry, Remote Sensing and Spatial Information Sciences, Volume II-5, 117-122

Gergont, K., Kierys, E. (2009). 3D - Scanning Technology in Forensic Science and Road Crash Investigations (MSc Diploma Thesisi), Kraków.

Luhmann T., Robson S., Kyle S., Boehm J. (2013). Close - Range Photogrammetry and 3D Imaging, De Gruyter Textbook

Markiewicz J., \& Turek A. (2014) The application of multitemporal aerial images in research on changes in spatial management. Przegląd Geodezyjny, 3-9.

Mousa W. (2014). Integration of Digital Photogrammetry and Terrestrial Laser Scanning for Cultural Heritage Data Recording, Verlag der Bayerischen Akademie der Wissenschaftenin Kommission beim Verlag S. H. Beck

Radziejewski R., (2013), Inventory of an industrial object with the use of terrestrial laser scanning, (MSc Diploma thesis), Warsaw University of Technology

Radziejewski R., Markiewicz J., \& Zawieska D.(2014) Diversity of photogrammetric approaches for multi-purpose applications, GeoConference on informatics, geoinformatics and remote sensing conference proceedings, Volume II Geodesy and Mine Surveying, 43-48.

Vosselman, G., Maas, H-G., (2012) Airborne and Terrestrial Laser Scanning, Whitles Publishing.

\section{Authors:}

\author{
Prof Dorota Zawierska ${ }^{1)}$, d.zawieska@gik.pw.edu.pl \\ Msc Jakub Markiewicz ${ }^{1)}$, j.markiewicz@gik.pw.edu.pl \\ 1) Faculty of Geodesy and Cartography \\ Warsaw University of Technology \\ PI. Politechniki 1, 00-661, Warsaw, Poland
}

